\title{
A Randomized, Double-Blind, Placebo- Controlled Trial on Intravenous Ibuprofen L-Lysine for the Early Closure of Nonsymptomatic Patent Ductus Arteriosus within 72 Hours of Birth in Extremely Low-Birth-Weight Infants
}

\author{
Jacob V. Aranda, M.D., Ph.D., F.R.C.P.C., ${ }^{1}$ Ronald Clyman, M.D., ${ }^{2}$ Blair Cox, M.D.., ${ }^{\dagger}$ \\ Bart Van Overmeire, M.D., Ph.D., 4 Paul Wozniak, M.D., 5 Ilene Sosenko, M.D., 6 \\ Waldemar A. Carlo, M.D.,' Robert M. Ward, M.D., 8 Robert Shalwitz, M.D.,9 \\ Geraldine Baggs, Ph.D., ${ }^{\prime}$ Anand Seth, Ph.D., 9 and Laszlo Darko, Ph.D. ${ }^{10}$
}

A multicenter, double-blind, randomized, placebo-controlled trial was conducted to evaluate the efficacy and safety of intravenous (IV) ibuprofen (L-lysine) for the early closure of nonsymptomatic patent ductus arteriosus (PDA) within 72 hours of birth in extremely low-birth-weight (ELBW) infants with evidence of ductal shunting by echocardiogram. Eleven sites enrolled 136 infants with nonsymptomatic early PDA (gestational age $<30$ weeks; body weight 500 to $1000 \mathrm{~g})$ to receive a 3 -day course $(10 \mathrm{mg} / \mathrm{kg}, 5 \mathrm{mg} / \mathrm{kg}$, and $5 \mathrm{mg} / \mathrm{kg})$ of IV ibuprofen $(n=68)$ or placebo $(n=68)$. Cardiac echocardiogram was performed on study days 1 and 14, and with rescue. Infants were followed to 36 weeks postconceptional age. Patient demographics, mean (standard deviation), were similar between ibuprofen and placebo: birth weight: $798.5 \mathrm{~g}$ (128.7) versus $797.3 \mathrm{~g}$ (132.8); gestational age: 26.1 weeks (1.3) versus 26.2 weeks (1.4); and age at first dose: 1.5 days (0.7). The intent-to-treat analysis of the primary endpoint, subjects rescued, died, or dropped through study day 14, was 21/68 (30.9\%) with ibuprofen and 36/68 (52.9\%) for placebo $(p=0.005)$. Death, intraventricular hemorrhage, necrotizing enterocolitis, daily fluid intake/output, liver function, bronchopulmonary dysplasia, and retinopathy of

\footnotetext{
${ }^{\dagger}$ Deceased.

${ }^{1}$ Pediatric Pharmacology Research Unit Network, The Children's Hospital of Michigan, Wayne State University, Detroit, MI; ${ }^{2}$ University of California, San Francisco, San Francisco, California; ${ }^{3}$ Department of Pediatrics, Neonatal-Perinatal Medicine Division, University of Texas, Southwestern Medical Center, Dallas, Texas; ${ }^{4}$ University of Antwerp, Antwerp University Hospital, Edegem, Belgium; ${ }^{5}$ University of California, San Diego, San Diego, California; ${ }^{6}$ Jackson Memorial Hospital, Miami, Florida; ${ }^{7}$ Division of Neonatology, University of Alabama at Birmingham, Birmingham, Alabama; ${ }^{8}$ Pediatric Neonatology, University of Utah Hospital, Salt Lake City, Utah; ${ }^{9}$ Abbott Laboratories, Columbus, Ohio; ${ }^{10}$ Farmacon IL, Inc., Stratford, Connecticut.
}

Address for correspondence and reprint requests: Jacob V. Aranda, M.D., Professor and Director of Neonatology, Pediatric Pharmacology Research Unit Network, The Children's Hospital of Brooklyn, State University of New York Downstate Medical Center, 450 Clarkson Avenue, Box 49, Brooklyn, New York City, NY 11023 (e-mail: jaranda@downstate.edu).

Am J Perinatol 2009;26:235-246. Copyright (C) 2009 by Thieme Medical Publishers, Inc., 333 Seventh Avenue, New York, NY 10001, USA. Tel: +1 (212) 584-4662.

Received: August 21, 2008. Accepted after revision: September 22, 2008. Published online: December 9, 2008.

DOI 10.1055/s-0028-1103515. ISSN 0735-1631. 
prematurity did not differ. A trend toward decreased periventricular leukomalacia by ibuprofen was noted. IV ibuprofen was effective and safe in the early closure of PDA in preterm neonates.

KEYWORDS: Ibuprofen, patent ductus arteriosus, premature newborn, cyclooxygenase inhibitors, randomized clinical trial

$\mathrm{P}_{\text {atent ductus arteriosus (PDA) is a common }}$ problem encountered in premature infants, especially those with respiratory distress syndrome. ${ }^{1,2}$ The incidence is inversely related to gestational age and birth weight, ${ }^{3,4}$ with 55 to $70 \%$ of infants born before 28 weeks' gestation and weighing $<1000 \mathrm{~g}$ at birth requiring treatment. ${ }^{5}$ A persistent $\mathrm{PDA}$ is usually diagnosed when the ductus fails to close spontaneously after 72 hours. ${ }^{6}$ The open ductus produces hemodynamic problems, which can lead to numerous clinical complications including congestive heart failure, intraventricular hemorrhage (IVH), necrotizing enterocolitis (NEC), bronchopulmonary dysplasia (BPD), and death. ${ }^{7,8}$

Medical therapy with cyclooxygenase inhibitors, particularly indomethacin, has been shown to be effective in ductal closure. Unfortunately, it has been associated with increased risk of renal failure, hematologic problems, NEC, decrease in cerebral blood flow $(\mathrm{CBF})$ velocity and bioenergetics. ${ }^{7-12}$ Furthermore, it is associated with a failure rate between $30 \%$ and 35\%, requiring retreatment or surgical closure of the ductus arteriosus. ${ }^{9,13,14}$

Ibuprofen is an alternative cyclooxygenase inhibitor available via the intravenous (IV) route. It has been effective in closing the ductus ${ }^{15-17}$ without reducing $\mathrm{CBF}^{18}$ or impacting intestinal ${ }^{15,19}$ or renal hemodyamics. ${ }^{20}$ Clinical trials have demonstrated equal efficacy versus indomethacin for ductal closure in treating a symptomatic PDA, with a more favorable renal safety profile and lack of effect upon CBF. ${ }^{17,18,21,22}$

PDA prophylaxis trials have shown that ibuprofen reduced the incidence of PDA, but failed to demonstrate any additional benefit over placebo, or in preventing intraventricular hemorrhage. ${ }^{23,24}$ The $60 \%$ spontaneous closure rate ${ }^{23}$ reported in these trials may lead practitioners to hold off treatment until clinical signs and symptoms appear several days after birth. Unfortunately, the delaying of therapy until hemodynamic symptoms of a persistent PDA appear, usually $>3$ to 6 days, increases the likelihood of developing PDArelated morbidities and may decrease the success of pharmacological closure. ${ }^{25}$

To date, there has been a lack of placebo-controlled data specific to the effectiveness or safety of early ibuprofen use ( $<72$ hours), in infants who are treated beyond the prophylactic period (12 hours after birth), but before a hemodynamically symptomatic PDA develops (3 to 9 days). In several prior studies, ibuprofen was given once a symptomatic PDA appeared, ranging from a few days to late in the first week after birth. Furthermore, many of the ibuprofen studies utilized an active comparator (indomethacin). Studies were also conducted in infants of older gestational age and with heavier birth weights than those currently being treated for a symptomatic PDA. ${ }^{26}$ This population difference is notable as spontaneous closure is inversely related to birth weight and gestational age, especially infants in under 28 weeks versus those over 29 weeks. ${ }^{27,28}$

As a result, this study aimed to determine the effect of early treatment with intravenous (IV) ibuprofen given to extremely low-birth-weight infants (ELBW) with a nonsymptomatic PDA in the first 72 hours of life compared with intravenous placebo, as the first such trial. The design was to test the hypothesis that ibuprofen given during this time period would accelerate and maintain ductal closure and, thereby, show a significant reduction in rescue therapy (indomethacin or surgical ligation). Furthermore, this trial, the first evaluation of ibuprofen L-lysine in the United States, was able to enroll infants of younger gestational age and lower birth weight (who are more commonly treated in North America) $)^{29,30}$ than the more mature infants enrolled in the earlier ibuprofen treatment trials.

\section{MATERIALS AND METHODS}

This is the first study in the United States to determine the efficacy and safety of the IV formulation of ibuprofen L-lysine in preterm newborns with nonsymptomatic PDA. This trial was a multicenter, randomized, placebo-controlled, double-blind parallel design study evaluation of IV ibuprofen using an intent-to-treat analysis to evaluate early closure of nonsymptomatic PDA within 72 hours of birth in ELBW infants. Eleven centers participated in this trial from March 2002 to March 2005 (Appendix 1). The protocol was approved by the Institutional Review Board for all participating sites, and by The Eunice Kennedy Shriver National Institute of Child Health and Human Development (NICHD) Pediatric Pharmacology Research Unit Network (PPRU).

\section{Inclusion-Exclusion Criteria}

All premature newborns born $\leq 30$ weeks' gestation admitted to the neonatal intensive care unit of the 
participating hospitals were eligible for this study if they met the inclusion criteria: (1) birth weight of 500 to $1000 \mathrm{~g}$; (2) < 72 hours postnatal age; (3) nonsymptomatic PDA with evidence of ductal shunting documented by echocardiogram; and (4) informed consent signed by parent/legal guardian.

Exclusion criteria included: (1) major congenital malformations and/or chromosomal anomalies; (2) proven congenital bacterial infection; (3) maternal antenatal nonsteroidal anti-inflammatory exposure $<72$ hours before delivery; (4) treatment with a steroid at anytime since birth; and (5) unremitting shock requiring high doses of vasopressors (i.e., inability to maintain mean arterial blood pressure appropriate for gestational age \pm 2 standard deviations [SDs] using volume and maximal vasopressor therapy as defined by the individual institution); (6) renal failure or oliguria defined as urine flow rate $<0.5 \mathrm{~mL} / \mathrm{kg} / \mathrm{h}$ in the 8 hours prior to randomization (anuria was acceptable if infant was within first 24 hours of life; (7) platelet count $<75,000 / \mathrm{mm}^{3}$; (8) clinical bleeding tendency (i.e., oozing from puncture sites); (9) expected survival $<48$ hours in the opinion of the attending neonatologist; and (10) participation in other clinical intervention trials unless approved by the medical director or study coordinator. For multiple births, no more than two of the infants could be enrolled.

\section{Sample Size, Randomization, and Drug Dosing}

Based on the assumptions of a $50 \%$ reduction in rescue rate in neonates with symptomatic PDA from $70 \%$ in the placebo group to $35 \%$ in the ibuprofen group, 120 evaluable infants ( $n=60$ per treatment group) were required to detect this difference with $90 \%$ power and $\alpha$ of 0.01 . Subjects were stratified into two birth weight categories: 500 to $750 \mathrm{~g}$ and 751 to $1000 \mathrm{~g}$. Following echocardiogram confirmation of a PDA, infants were randomized into two groups to receive either ibuprofen (L-lysine formulation) or placebo (normal saline) within 72 hours of birth. The coded vials of study drug or placebo contained indistinguishable colorless solutions dispensed by the blinded research pharmacists of the participating sites. Central randomization (ClinPhone Plc., Nottingham, UK) was implemented using a dynamic allocation method of biased coin randomization, ${ }^{31}$ balancing within birth weight, within each site, and in the study overall. Placebo or ibuprofen $(10 \mathrm{mg} / \mathrm{mL})$ was given intravenously for 10 minutes using $10 \mathrm{mg} / \mathrm{kg}$ loading dose followed by $5 \mathrm{mg} / \mathrm{kg} / \mathrm{d}$ on the second and third study days, using umbilical venous catheter or peripheral IV site.

\section{Outcome Assessments and Procedures}

Outcome assessments were collected from enrollment through study day 14. Follow-up outcomes were col- lected at 36 weeks postconceptional age ( \pm 7 days) or at transfer from facility. Adverse events were collected through 30 days after the last administration of study drug.

The primary outcome was the presence of a symptomatic PDA requiring rescue with indomethacin or surgery as determined by the attending neonatologist and staff. This endpoint was selected based on the assumption that a clinically significant PDA requires intervention with indomethacin or surgery and is in accord with the Food and Drug Administration (FDA) regulatory requirement of providing a clinically significant outcome beyond ductal closure for this placebo-controlled study. As this study involved an intentto-treat analysis, all dropouts and deaths were included as part of this primary endpoint.

The presence of a clinically significant PDA was determined by three or more clinical and physical findings of symptomatic ductus (bounding pulses, hyperdynamic precordium, pulmonary edema, increased radiographic cardiac silhouette, systolic murmur), confirmed by an echocardiogram, and requiring intervention.

Echocardiograms were done within the first 72 hours of age, prerandomization, and at study day 14 ( \pm 1 day). Additional echocardiogram(s) were performed if rescue therapy was considered for symptomatic PDA. Echocardiography using color Doppler echocardiography (Sonos 5500 or Sonos 1500 imaging system; Hewlett-Packard, Andover, MA, or Ultramark 4 Plus; Advanced Technology Laboratories, Bothell, WA with a $7.5-\mathrm{MHz}$ or $10-\mathrm{MHz}$ transducer) was performed by cardiologists unaware of infants' treatment. The purpose of the echocardiography was to evaluate patency of the ductus and shunting at the time of inclusion, at anytime rescue therapy with indomethacin or surgery was considered, and at study day 14. M mode was used to calculate the shortening fraction and to measure left ventricular internal diameter in diastole (left ventricle $[\mathrm{LV}])$. A two-dimensional parasternal long-axis view was obtained for assessment of the diameter of the left atrium (LA) in systole and aortic root diameter (AO). Ratios of $\mathrm{LA} / \mathrm{AO}$ and $\mathrm{LV} / \mathrm{AO}$ were calculated off-line from these diameters. A twodimensional ductal view with color Doppler was performed to assess the ductal shunting and minimum diameter of the PDA.

The guideline for a hemodynamically significant PDA was the presence of at least two of the following three parameters: (1) LA/AO ratio of $>1.4: 1$; (2) LV/ $\mathrm{AO}$ ratio of $>2.1: 1$; and/or (3) narrowest ductal diameter $>1.5 \mathrm{~mm}$. Cardiac echocardiogram provides more accurate assessment of PDA; however, a clinically based decision by the neonatologist was deemed more practical in the clinical setting using criteria noted above. 


\section{Safety Considerations and Assessments}

Subjects with symptomatic PDA at any time during the study could receive rescue treatment at the discretion of the investigator/attending neonatologist, provided the infant met criteria for medical or surgical intervention. No data on potential synergistic interactions of ibuprofen and indomethacin in newborns were known; therefore, if rescue therapy with indomethacin was indicated, it was administered at least 24 hours from last dose of study drug, based on plasma ibuprofen half-life of $\sim 23$ hours. ${ }^{32}$

Secondary outcomes were related to safety. NEC was graded using Bell's classification. ${ }^{33}$ Retinopathy of prematurity (ROP) was evaluated as per standard of care.

Respiratory effects were closely monitored and evaluated. Pulmonary hemorrhage and persistent pulmonary hypertension in newborns (PPHN) were tracked at study day 14. PPHN was diagnosed after severe hypoxemia was observed. This was defined as hypoxic respiratory failure with an oxygenation index (mean airway pressure $\left.\times \mathrm{FiO}_{2} \times 100 / \mathrm{PaO}_{2}\right)(\mathrm{OI}) \geq 15$ on two arterial gases at least 15 minutes apart and $\leq 12$ hours apart. Because of the brief duration of these hypoxemic episodes $(20 \mathrm{~min})^{34}$ and their good response to inhaled nitric oxide, no echocardiograms were obtained for PPHN diagnosis. Respiratory data, including $\mathrm{FiO}_{2}$ (inspired oxygen) and method of ventilatory support, were collected at baseline and study days 4 and 14. BPD was the need for supplemental oxygen at 28 postnatal days or 36 weeks postconceptional age.

IVH was a secondary outcome for safety and was not an exclusion criteria for the study. Although ibuprofen has been shown to improve $\mathrm{CBF}$ autoregulation in neonatal piglets, ${ }^{35}$ at least two clinical trials showed no effect on the risk of IVH in preterm neonates. ${ }^{24,36}$ Moreover, nonsteroidal anti-inflammatory drugs, like indomethacin, do not worsen or cause extension of an existing $\mathrm{IVH}^{37}$

IVH was evaluated by cerebral ultrasounds. For study purposes, ultrasounds were performed as standard of care and were performed closest to study days 4 and 14. As such, these ranged from day of life 1 to 24 . The lesions were classified as described by Papile et al. ${ }^{38}$ All cranial ultrasounds were read by radiologists of participating centers. Periventricular leukomalacia (PVL) was graded by the characteristics of periventricular white matter on sonography. ${ }^{39}$

Serum laboratories and fluid status were monitored during the study period. Serum bilirubin, creatinine, electrolytes, and blood urea nitrogen were recorded daily from study days 1 through 6 . Daily fluid intake and output for 24-hour intervals were recorded from birth through day 10 of life. Complete blood counts were collected at baseline and study day 4. Liver enzymes including serum glutamic pyruvic transaminase (SGPT), serum glutamic oxaloacetic transaminase (SGOT), and serum gamma glutamyl transferase (SGGT) were collected at baseline and at study day 14 .

Plasma samples for ibuprofen concentrations were obtained at 1 hour ( \pm 30 minutes) after the first dose; at 24 hours ( \pm 1 hour) just prior to dose 2 ; at 48 hours (prior to dose 3), and at 120 hours. Plasma ibuprofen assays by high-pressure liquid chromatography were performed at the conclusion of the clinical trial when blinding was broken.

\section{Data Analysis}

Data were analyzed using the intent-to-treat principle. The primary endpoint comprised all subjects who died, dropped out, or required rescue. Analysis involved logistic regression with factors for treatment and site and utilized SAS ${ }^{\circledR}$ software and procedures (SAS Corporation, Cary, NC). Furthermore, a confirmatory analysis was performed using logistic regression with covariates: birth weight (500 to $750 \mathrm{~g}, 751$ to $1000 \mathrm{~g}$ ), gestational age $(<28, \geq 28$ weeks), gender, use or nonuse of highfrequency oscillatory ventilation, and maximum weight loss during the first 7 days of life. Because this was a Phase III confirmatory trial, a $p$ value of $<0.01$ for the primary outcome was designated as significant. Other statistical analyses of efficacy parameters included the Wilcoxon rank-sum tests or Cochran-Mantel-Haentzel tests stratified by site and repeated measures analyses. Kaplan-Meier survival curves were presented for survival data with statistical significance tested utilizing a logrank test.

Adverse event data were evaluated via the Data Safety Monitoring Committee (DSMC), composed of experts in neonatology, pediatric cardiology, pharmacology, epidemiology, and biostatistics (Appendix 1). A mandatory review of adverse event data was conducted after $25 \%, 50 \%$, and $75 \%$ of infant recruitment. Committee members received blinded summary data grouped by treatment codes. A formal summary of each safety review was written by the committee and incorporated in study file. Communications with DSMC was through a designated biostatistician for the study.

\section{RESULTS}

\section{Subject Disposition and Characteristics}

Nine hundred forty-four very low-birth-weight neonates were screened for the study. Of these infants, 749 were considered not eligible because of maternal tocolysis with indomethacin, enrollment in other studies, or parental refusal to give informed consent. From the remaining 195 subjects who gave informed consent, 59 failed to meet inclusion and/or exclusion criteria or did not have a patent ductus on the initial cardiac echocardiogram. Therefore, 136 preterm newborns 
Table 1 Demographics

\begin{tabular}{|c|c|c|}
\hline Variable & Ibuprofen* $(n=68)$ & Placebo $(n=68)$ \\
\hline Birth weight (g), mean (SD) & $798.5(128.7)$ & $797.3(132.8)$ \\
\hline Gestational age (wk), mean (SD) & $26.1(1.3)$ & $26.2(1.4)$ \\
\hline \multicolumn{3}{|l|}{ Gender, $n(\%)$} \\
\hline Male & $32(47)$ & $37(54)$ \\
\hline Female & $36(53)$ & $31(46)$ \\
\hline \multicolumn{3}{|l|}{ Ethnicity, $n(\%)$} \\
\hline Caucasian & $23(34)$ & $18(26.5)$ \\
\hline Black & $17(25)$ & $18(26.5)$ \\
\hline Hispanic & $21(31)$ & $28(41)$ \\
\hline Asian/Pacific Islander & $1(1.5)$ & $2(3)$ \\
\hline Other & $6(9)$ & $2(3)$ \\
\hline Apgar score, 1 minute (median, IQR) & $4.0(2.0), n=67$ & $4.0(2.0), n=67$ \\
\hline Apgar score, 5 minutes (median, IQR) & $7.0(2.0), n=67$ & $7.0(2.0), n=68$ \\
\hline Maternal age, mean (SD) & $28.0(6.6)$ & $27.8(6.6)$ \\
\hline Maternal steroid use, $n / N(\%)$ & $51 / 67(76.1)$ & 48/68 (70.6) \\
\hline Age of first dose of study drug, mean (SD) & $1.5(0.74)$ & $1.4(0.73)$ \\
\hline Baseline neurological exam abnormal, $n / N(\%)$ & 6/68 (8.8) & $4 / 68(5.9)$ \\
\hline Baseline apnea, $n / N(\%)^{\dagger}$ & $15 / 68(22)$ & 13/68 (19) \\
\hline Mechanical ventilation, $n / N(\%)$ & $47 / 68(69)$ & $46 / 68(67)$ \\
\hline
\end{tabular}

*No significant difference between treatment and placebo: $p>0.05$.

† Defined as no respiratory effort $>15 \mathrm{~s}$, with bradycardia (heart rate $<100$ beats/min).

$\mathrm{SD}$, standard deviation; IQR, interquartile range.

were enrolled and randomized to receive either placebo or study drug.

Placebo- and ibuprofen-treated groups were similar in demographic characteristics, including birth weight, gestational age, ethnicity (Table 1). Subjects were enrolled within 72 hours after birth, and mean ages and standard deviation of the first dose were 1.5 (0.74) and 1.4 (0.73) days for ibuprofen and placebo group, respectively.

\section{Efficacy Analysis}

In this study of early closure of nonsymptomatic PDA within 72 hours of birth in ELBW infants, efficacy outcomes have been depicted in Figs. 1 and 2. Based upon the intent-to-treat analysis of the primary endpoint, the ibuprofen group had a significantly lower proportion of infants who died, dropped out, or required rescue $(21 / 68 ; 30.9 \%)$ as compared with the placebo group $(36 / 68 ; 52.9 \% ; p=0.005)$ for symptomatic PDA on or before study day 14 as depicted in Fig. 1. This significant difference was even more pronounced when adjusted for covariates $(p=0.0014)$, including birth weight, gestational age, gender, maximum weight loss, use of oscillatory ventilation, and site.

Excluding those who died before study day 14, a significantly lower proportion of infants needed rescue (Fig. 2) in the ibuprofen group compared with the placebo group $(25.0 \%$ versus $48.5 \%, p=0.003)$. In addition, the mean (SD) age of first rescue treatment was 8.7 (3.8) versus 6.9 (3.2) days for ibuprofen and placebo, respectively. Interestingly, the proportion of infants requiring rescue with indomethacin on or prior to study day 14 who were surgically ligated (Fig. 2) was not significantly different between the ibuprofen (8/17; $47.1 \%)$ and the placebo group $(9 / 33 ; 27.3 \%)$.

With respect to rescue after study day 14, 5 (7.4\%) ibuprofen infants and 4 (5.9\%) placebo patients were rescued. The mean ages were 19 and 18 days in the

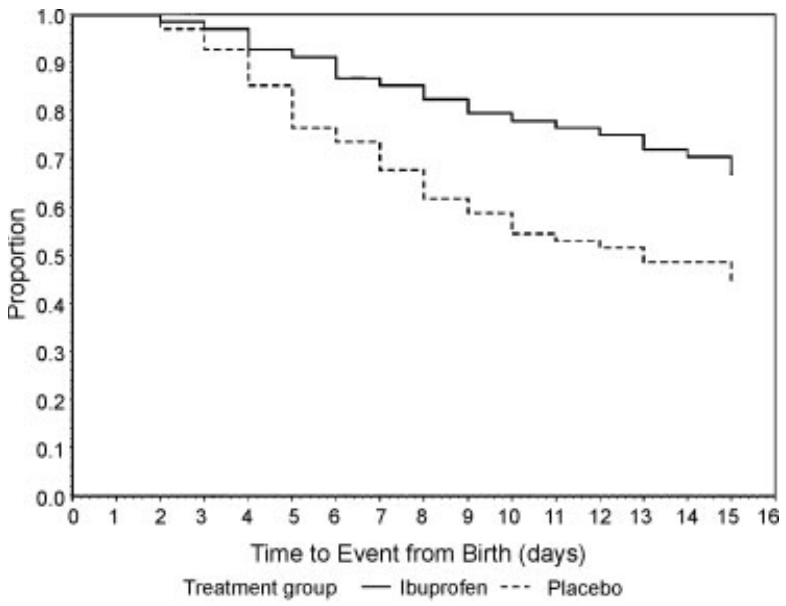

Figure 1 Kaplan-Meier curve showing the proportions of study participants who did not have any intervention, who dropped out, or who died. Broken line represents the placebo group; solid line represents the ibuprofen group. Log ranksum test $p$ value $=0.006$. 


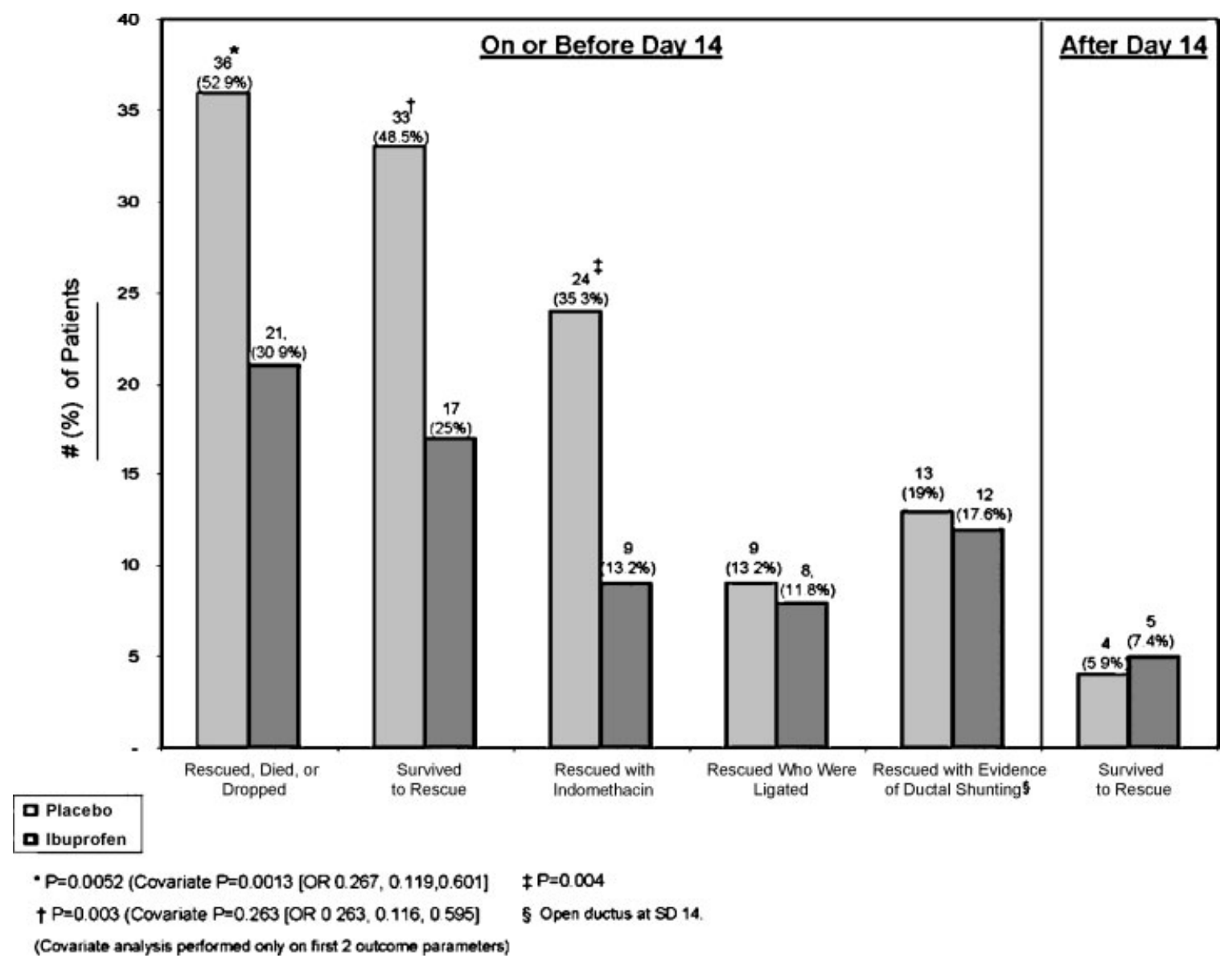

Figure 2 Summary of efficacy outcomes.

ibuprofen and placebo groups, respectively. Of these patients, three ibuprofen patients and one placebo $\mathrm{pa}^{-}$ tient subsequently underwent surgery, and one patient in each group died due to NEC. Neither gestational age nor birth weight was related to the rates of rescue (Table 2). Placebo treatment was the only factor statistically associated with an increased rescue outcome (Table 2).

Of the 30 infants (40\%) in the placebo group who were not accounted for as either deaths, dropouts, or rescued patients either before, on, or after study day 14 , 20 (29\%) did not present with evidence of ductal shunting and, hence, were identified as having undergone spontaneous closure.

\section{Safety Analysis and Serum Drug Concentrations}

Safety endpoints have been summarized in Table 3. Higher urine flow rate was noted in the ibuprofen group at day 9 , reflecting the effect of indomethacin rescue in a greater proportion of infant in the placebo group (Fig. 3A). Mean serum creatinine, within upper normal range in both groups, was significantly higher in the ibuprofen group on study days 3 and 4 (Fig. 3B); however, when adjusted for baseline, these differences disappear. Blood urea nitrogen was not significantly different between the placebo and ibuprofen groups (Fig. 3C) at any time point. Fluid intakes were also similar in both groups (Fig. 3D).

Table 2 Factors Associated with Rescue in Preterm Newborns with Patent Ductus Arteriosus

\begin{tabular}{llll}
\hline Effect & $\begin{array}{l}\text { Adjusted } \\
\text { Odds Ratio }\end{array}$ & $\begin{array}{l}\mathbf{9 5 \%} \text { Confidence } \\
\text { Interval }\end{array}$ & $\boldsymbol{p}$ Value \\
\hline Treatment: ibuprofen vs. placebo & 0.27 & $(0.12,0.60)$ & 0.001 \\
Birth weight: $500-750$ vs. $751-1015$ & 0.90 & $(0.38,2.17)$ & 0.817 \\
Gestational age: $<28$ vs. $\geq 28$ & 2.38 & $(0.66,8.58)$ & 0.187 \\
Sex & 1.05 & $(0.47,2.36)$ & 0.904 \\
High-frequency oscillatory ventilation use: no vs. yes & 0.40 & $(0.15,1.06)$ & 0.065 \\
Maximum weight loss during the first 7 d of life (per 100-g loss) & 0.49 & $(0.22,1.08)$ & 0.076 \\
\hline
\end{tabular}


Table 3 Safety Outcomes and Adverse Events

\begin{tabular}{|c|c|c|}
\hline $\begin{array}{l}\text { Safety Outcomes and } \\
\text { Adverse Events* }\end{array}$ & $\begin{array}{l}\text { lbuprofen, } \\
n / N(\%)\end{array}$ & $\begin{array}{l}\text { Placebo, } \\
n / N(\%)\end{array}$ \\
\hline NEC & 9/65 (13.8) & 9/65 (13.8) \\
\hline \multicolumn{3}{|c|}{$\begin{array}{l}\text { Stages of NEC (highest } \\
\text { identified from all sources) }\end{array}$} \\
\hline None & $56 / 65(86.2)$ & $56 / 65(86.2)$ \\
\hline Pre-NEC & $2 / 65(3.1)$ & $2 / 65(3.1)$ \\
\hline Definite & $3 / 65$ (4.6) & $1 / 65(1.5)$ \\
\hline Advanced & $4 / 65(6.2)$ & $6 / 65(9.2)$ \\
\hline $\mathrm{IVH}$ & 25/67 (37.3) & $25 / 67(37.3)$ \\
\hline \multicolumn{3}{|l|}{ Worst reported IVH grade } \\
\hline None & $42 / 67(62.7)$ & $42 / 67(62.7)$ \\
\hline Grade I & 6/67 (9.0) & $7 / 67(10.4)$ \\
\hline Grade II & 8/67 (11.9) & 7/67 (10.4) \\
\hline Grade III & 8/67 (11.9) & 8/67 (11.9) \\
\hline Grade IV & $3 / 67$ (4.5) & $3 / 67$ (4.5) \\
\hline Pulmonary hemorrhage & $1 / 68(1.5)$ & $4 / 68(5.9)$ \\
\hline Pulmonary hypertension & $2 / 68(2.9)$ & $1 / 68(1.5)$ \\
\hline ROP & 40/65 (61.5) & $33 / 64(51.6)$ \\
\hline \multicolumn{3}{|l|}{ Stages of ROP } \\
\hline None & 25/65 (38.5) & $31 / 64(48.4)$ \\
\hline Stage 1 or 2 & $34 / 65$ (52.3) & 28/64 (43.8) \\
\hline $\begin{array}{l}\text { Stage } 3 \text { or } 4 \text { (no stage } \\
4 \text { were observed) }\end{array}$ & $6 / 65(9.2)$ & $5 / 64(7.8)$ \\
\hline ROP plus disease & $4 / 65(6.2)$ & $3 / 64(4.7)$ \\
\hline \multicolumn{3}{|l|}{ BPD } \\
\hline $\mathrm{O}_{2}$ need at $28 \mathrm{~d}$ & $58 / 65(89.2)$ & $53 / 65(81.5)$ \\
\hline $\begin{array}{l}\mathrm{O}_{2} \text { need at } 36 \mathrm{wk} \\
\text { corrected age }\end{array}$ & 42/46 (91.3) & $48 / 52$ (92.3) \\
\hline PVL & $0 / 65(0.0)^{\dagger}$ & $4 / 65(6.2)$ \\
\hline Death & 8/68 (11.8) & 10/68 (14.7) \\
\hline
\end{tabular}

${ }^{*}$ Chi-square test based on logistic regression controlling for sites. ${ }^{\dagger} p=0.0587$.

NEC, necrotizing enterocolitis; IVH, intraventricular hemorrhage; $R O P$, retinopathy of prematurity; $B P D$, bronchopulmonary dysplasia; $\mathrm{PVL}$, periventricular leukomalacia.

With respect to complications and adverse events, there were no differences in the proportion of babies who developed IVH, NEC, BPD, PPHN, or ROP between groups. None of the ibuprofen-treated infants had PVL, whereas four newborns in the placebo group had PVL diagnosed by head ultrasound at 36 weeks or prior to discharge $(p=0.059)$.

BPD at 28 days or at 36 weeks corrected age was higher than the usual average rates of $\sim 40 \%$. The high rate of oxygen use in both groups reported in this trial is likely explained by the broad definition of oxygen use and the low weight and gestational age of the population. Ventilator use was high in both groups on study day $1(\sim 80 \%)$ but decreased to $\sim 57 \%$ in both groups by study day 14. Many of the infants continued to require supplemental inspired oxygen, albeit of low concentrations via nasal cannula (e.g., $22 \%$ inspired oxygen).
Liver function tests were evaluated for both groups. SGPT values did not vary between ibuprofen and placebo, respectively, at baseline $(15.8 \mathrm{U} / \mathrm{L}$ versus $14.3 \mathrm{U} / \mathrm{L}$ ) and study day 14 (14.9 versus 15.2). However, SGGT decreased from baseline to study day 14 for both ibuprofen $(91.8 \mathrm{U} / \mathrm{L}$ to $51.0 \mathrm{U} / \mathrm{L})$ and placebo (103.0 U/L to $47.9 \mathrm{U} / \mathrm{L})$. SGOT also decreased for both Ibuprofen (54.4 U/L to $27.6 \mathrm{U} / \mathrm{L})$ and placebo $(55.9 \mathrm{U} / \mathrm{L}$ to $27.9 \mathrm{U} / \mathrm{L}$ ). Changes from baseline and difference between treatments were not deemed to be significant.

Mean plasma concentrations ( \pm SD) of ibuprofen at $1,24,48$, and 120 hours after the first dose were: 34.7 (9.0), 24.2 (7.6), $27.3(14.2)$, and $13.2(11.5) \mathrm{mg} / \mathrm{L}$, respectively. There were no correlations between plasma concentration and primary outcome, PDA closure, or adverse effects.

\section{DISCUSSION}

The purpose of this study was to evaluate the effectiveness and safety of early closure of nonsymptomatic PDA within 72 hours of birth in ELBW infants with evidence of ductal shunting by echocardiogram with IV ibuprofen. In contrast to other ibuprofen studies that used ductal closure as the primary endpoint, this study was unique in its design to address the specific question as to whether treatment versus no treatment (placebo) during the early preterm setting ( $<72$ hours of birth) imparted a clinically meaningful and statistically significant benefit, preventing the need for rescue with surgery or indomethacin.

This was the first study in the United States conducted with ibuprofen L-lysine. This is important because birth weights and gestational ages tend to be lower in infants than in other countries. In this study, the mean birth weight was $\sim 798 \mathrm{~g}$ and gestational age was $\sim 26$ weeks. This contrasts infant characteristics observed in other studies with ibuprofen conducted internationally where the mean weights ranged from 900 to $1200 \mathrm{~g}$ and gestational ages were $\sim 29$ weeks. These characteristics represent important population differences that have significant clinical implications. It is important to recognize that the rate of spontaneous closure of the ductus without treatment is inversely related to birth weight and gestational age, especially in infants under the age of 28 weeks versus those over 29 weeks. $^{27,28}$

Placebo, rather than indomethacin, was used for comparison to address the specific question: does ibuprofen reduce the need for rescue therapy by early recognition and treatment of an asymptomatic, but early identifiable PDA? This study also met an FDA regulatory requirement for a placebo-controlled study that was ethical and allowed for the evaluation of a clinically meaningful endpoint beyond ductal closure such as rescue rates. Furthermore, this study was designed to 


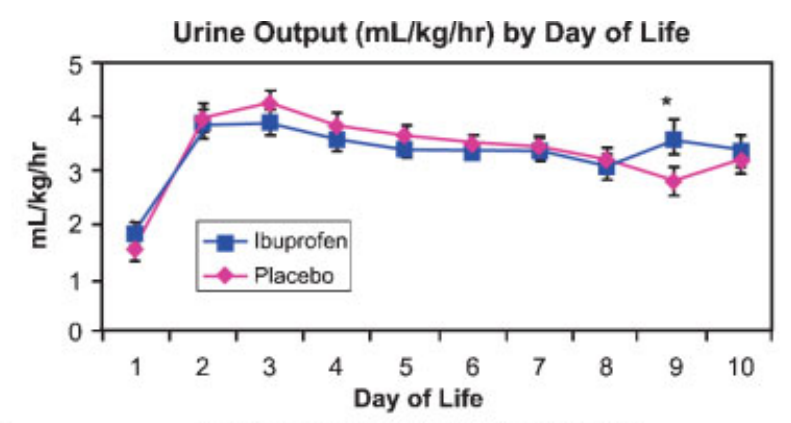

A

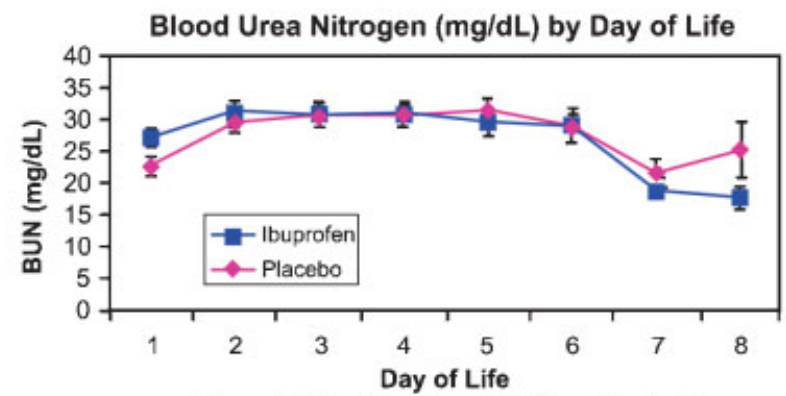

Day of Life 8 had $n<10$ (not tested)
Creatinine $(\mathrm{mg} / \mathrm{dL})$ by Day of Life

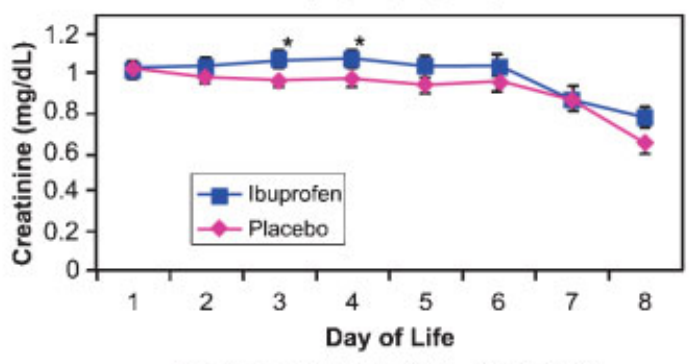

Ibuprofen > Placebo, $\mathrm{p} \leqq 0.0058$

B

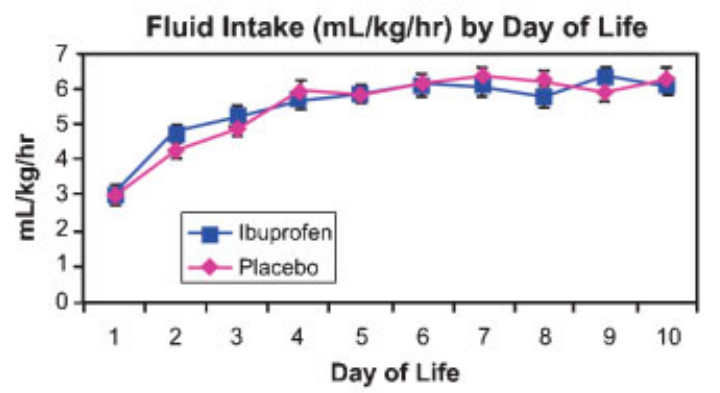

D

Figure 3 Renal effects of ibuprofen. (A) Effect on urine output. There was a significantly lower urine output at day 9 in the placebo group $(p<0.02$ ) compared with the ibuprofen group. More subjects received indomethacin in the placebo group, which may have been associated with this lower renal output. (B) Effect on serum creatinine. Mean serum creatinine levels were below $1.1 \mathrm{mg} / \mathrm{dL}$ in both groups. A significantly higher serum creatinine level was noted in the ibuprofen group $(p<0.05)$ at days 3 and 4; however, these differences disappeared when corrected for baseline values. (C) Effect on blood urea nitrogen. No differences were noted except at day 8 when values in ibuprofen group were lower than placebo. (D) Fluid intake of the placeboand ibuprofen-treated groups. No differences were noted between the two groups.

answer questions from some practitioners who believe spontaneous closure will occur and will wait on active treatment until clinical symptoms present. Results from this trial indicate that, in this ELBW population, spontaneous closure rates were no $\leq 30 \%$. Moreover, closure of early asymptomatic PDA, outside of the prophylactic setting with indomethacin, has not been robustly evaluated and remains a matter of debate as it is also associated with a failure rate between $30 \%$ and $35 \%$, requiring retreatment or surgical closure of the ductus arteriosus. ${ }^{9}$ This is the subject of a currently ongoing large clinical trial in Australia (DETECT Trial) with indomethacin targeting asymptomatic patients who are between 6 and 12 hours of age and present with an open ductus. ${ }^{30}$ Thus, the need for a placebo control and the endpoint of rescue were determined to be real and clinically relevant.

Ibuprofen has been extensively studied. Previous trials that compared the clinical effects of ibuprofen versus an active treatment, indomethacin, ${ }^{17,25,40}$ have shown that ibuprofen is effective in PDA closure. Many of these studies, however, have evaluated treatment in different populations (prophylaxis, $>72$ hours after birth, different gestational age and birth weight profiles) or have been direct comparison versus an active treatment. No study has been published previously that specifically addressed the question regarding the clinical benefit of early medical therapy with a cyclooxygenase inhibitor versus placebo.

The efficacy results from this trial demonstrated that early treatment with ibuprofen of a confirmed PDA in infants $<72$ hours of birth can significantly decrease the need for further pharmacological or surgical intervention as compared with placebo. In addition to the significant reduction in the primary outcome, there was also a significant difference in the number of infants who had a completely closed ductus $(0 \mathrm{~mm})$ in the ibuprofen group versus placebo by study day 14 (69\% versus 33\%, $p=0.046)$, indicating that early therapy with ibuprofen versus placebo produces a meaningful clinical benefit in the treatment of a medically identifiable, but asymptomatic PDA. From a safety vantage point, the ibuprofen and the placebo groups did not significantly differ in rates of neonatal morbidities, including IVH, NEC, BPD, PPHN, and ROP.

Ibuprofen plasma concentrations were evaluated at $1,24,48$, and 120 hours after the first dose to determine any correlation with efficacy outcome or adverse events. The highest mean concentration $( \pm \mathrm{SD})$ was $34.7 \mathrm{mg} / \mathrm{L}(0.9)$, which was 1 hour after dosing and was significantly below levels deemed to be of clinical concern. There was no correlation of mean blood 
level versus resultant outcomes or appearance of adverse events; further work is planned to evaluate the pharmacokinetic profile of ibuprofen dosing from this study.

The data from this study are consistent with previous studies $^{16}$ and others ${ }^{25,36,41}$ that ibuprofen L-lysine, given during the first 3 days of life, significantly increases PDA closure. In addition, our study supports others that IV ibuprofen decreases PDA with minimal effect on renal function. ${ }^{16,25,41}$ However, a placebocontrolled PDA prevention study utilizing a different formulation (ibuprofen trishydroxymethylaminomethane) noted an increased risk of NEC, gastrointestinal perforation, $\mathrm{PPHN}$, and a higher proportion of neonates with elevated creatinine with IV ibuprofen, when given within 6 hours of birth. ${ }^{34,42}$ It should be noted that this study population was not screened for the presence of a PDA prior to therapy, and therefore, some patients were likely exposed to drug when they did not need it. Indeed, two of the three patients reported to have experienced PPHN in the trial by Gournay and colleagues $^{42}$ were found to have a constricted ductus and no shunt. These authors recommended an "early curative ibuprofen" regimen, which is similar to, and supportive of, the conclusions from the trial reported here.

In the present study, there were no differences noted in the incidence of NEC, gastrointestinal perforation, or PPHN between the ibuprofen and placebo groups. Feeding practices, not controlled in this study, differed between participating centers; some babies had no enteral feedings, although $\sim 80 \%$ of others were given trophic or feeding as per institutional standard of care practice. Studies on ibuprofen and indomethacin effects on mesenteric and renal blood flow show that ibuprofen does not alter blood flow 30 minutes after treatment, but increases it 120 minutes after treatment. ${ }^{22}$ In contrast, indomethacin has been shown to significantly reduce mesenteric and renal blood flow velocity 30 minutes after administration, without returning to pretreatment values within 120 minutes. This suggests that continued feeding may be acceptable with ibuprofen, although further controlled studies are needed to clarify this issue.

The minimal effects upon renal function, shown in this trial and others, ${ }^{24,25}$ are a reflection of ibuprofen's mild effect on renal prostaglandins. Ibuprofen treatment of neonatal rats in the first 3 postnatal days had minimal effects on renal prostaglandin E, F2a, 6 keto-prostaglandinGF1a, and thromboxane B2. ${ }^{43}$

There was a trend toward a decrease in PVL by head ultrasounds at 36 weeks, which has not been noted in previous studies. ${ }^{24}$ This observation is intriguing and bears further scrutiny as few pharmacologic agents with the exception of inhaled nitric oxide and caffeine have been shown to decrease PVL in preterm newborns. ${ }^{44,45}$ In previous studies, ibuprofen showed no effect on cerebral $\mathrm{CO}_{2}$ vasoreactivity, $\mathrm{CBF}$ velocities, and cytochrome c reductase levels, ${ }^{18,21}$ but the role of inflamma- tion on the pathogenesis of brain injury has been recognized recently. ${ }^{46,47}$

\section{CONCLUSION}

In summary, in this study of early closure of nonsymptomatic PDA within 72 hours of birth in ELBW infants, the treatment of echocardiogram-confirmed but asymptomatic PDA with IV ibuprofen L-lysine can significantly decrease the need for further pharmacological or surgical intervention as compared with placebo. In this study, IV ibuprofen L-lysine was associated with significantly lower rates of rescue, death, and dropouts, as compared with placebo. Furthermore, there was no difference in complications including IVH, NEC, $\mathrm{BPD}, \mathrm{ROP}$, or PPHN, indicating safety and efficacy of ibuprofen for early closure of PDA. Moreover, ibuprofen L-lysine had minimal effect on renal function and did not unduly decrease urine flow rate. The intriguing trend toward reduction of PVL with ibuprofen L-lysine requires further examination.

\section{ACKNOWLEDGMENTS}

The authors express their appreciation to the parents who consented to have their babies participate in the study; the nurses, neonatologists, and pharmacists who ensured excellent care for the study participants; the research nurse coordinators; and Jackie Parker, Ginger Steinhilber, Ronald Thomas, and all the members of the IV Ibuprofen Clinical Trials Team.

Supported by National Institutes of Health grant 5-U01HD-37261-01.

Funding and Study Sponsor: Ross Abbott Laboratories, Columbus, Ohio in collaboration with the NICHD Pediatric Pharmacology Research Unit Network. Thanks to members of the Ross Abbott Laboratories team. Study was registered with clinicaltrials.gov at www.clinicaltrials.gov; ID \#: NCT00440804. In memory of collaborators who have passed on, Laszlo Darko, Ph.D. and Blair Cox, M.D.

\section{DISCLOSURES}

Jacob V. Aranda, M.D., received honoraria for serving as educational consultant for Ross Abbott (December 2005) and Ovation Laboratories (2006-2008). He also served as unpaid (pro bono) and paid consultant (honoraria) on pediatric drug development for various pharmaceutical companies and clinical drug trials performed with the NICHD Pediatric Pharmacology Research Unit Network including Aventis, Merck, Purdue, Johnson and Johnson, Farmacon, Pfizer, and Bristol Myers Squibb-Mead Johnson.

Robert M. Ward, M.D., received an honorarium for serving as educational consultant for Ovation 
Laboratories in 2007. He has also served as an unpaid and paid consultant on pediatric drug development for various pharmaceutical companies including Wyeth Pharmaceuticals, Abbott Laboratories, Farmacon, McNeil Consumer Group, and Johnson \& Johnson during 2005.

Robert Shalwitz, M.D., was the Medical Director of Abbott Nutritionals during the trial.

Geraldine Baggs, Ph.D., and Anand Seth, Ph.D., are senior clinical research scientists at Abbott Nutritionals.

L. Darko, Ph.D. (deceased), was the CEO of Farmacon IL, Inc.

\section{REFERENCES}

1. Hammerman C. Patent ductus arteriosus. Clin Perinatol 1995;22:457-477

2. Krueger E, Mellander M, Bratton D, Cotton R. Prevention of symptomatic patent ductus arteriosus with a single dose of indomethacin. J Pediatr 1987;111:749-754

3. Dudell GG, Gersony WM. Patent ductus arteriosus in neonates with severe respiratory distress syndrome. J Pediatr 1984;104:915-920

4. Rubaltelli FF, Dani C, Reali MF, et al. Acute neonatal respiratory distress in Italy: a one-year prospective study. Acta Paediatr 1998;87:1261-1268

5. Hammerman C, Kaplan M. Comparative tolerability of pharmacological treatment for patent ductus arteriosus. Drug Saf 2001;24:537-551

6. Clyman RI. Ibuprofen and patent ductus arteriosus. N Engl J Med 2000;343:728-730

7. Cotton RB, Stahlman MT, Bender HW, et al. Randomized trial of early closure of symptomatic patent ductus arteriosus in small preterm infants. J Pediatr 1978;93:647-651

8. Gersony WM, Peckham GJ, Ellison RC, Miettinen OS, Nadas AS. Effects of indomethacin in premature infants with patent ductus arteriosus: results of a national collaborative study. J Pediatr 1983;102:895-906

9. Cooke L, Steer P, Woodgate P. Indomethacin for asymptomatic patent ductus arteriosus in preterm infants. Cochrane Database Syst Rev 2003;(2):CD003745: DOI: 10.1002/ 14651858.CD003745

10. Fowlie PW, Davis PG. Prophylactic intravenous indomethacin for preventing mortality and morbidity in preterm infants. Cochrane Database Syst Rev 2006;(3): CD000174. DOI: $10.1002 / 14651858 . C D 000174$

11. Yeh TF, Luken JA, Thalji A, Raval D, Carr I, Pildes RS. Intravenous indomethacin therapy in premature infants with persistent ductus arteriosus-a double-blind controlled study. J Pediatr 1981;98:137-145

12. Rennie JM, Doyle J, Cooke RW. Early administration of indomethacin to preterm infants. Arch Dis Child 1986;61: 233-238

13. Narayanan M, Cooper B, Weiss H, Clyman RI. Prophylactic indomethacin: factors determining permanent ductus arteriosus closure. J Pediatr 2000;136:330-337

14. Keller RL, Clyman RI. Persistent Doppler flow predicts lack of response to multiple courses of indomethacin in premature infants with recurrent patent ductus arteriosus. Pediatrics 2003;112(3 Pt 1):583-587
15. Coceani F, White E, Bodach E, Olley PM. Age-dependent changes in the response of the lamb ductus arteriosus to oxygen and ibuprofen. Can J Physiol Pharmacol 1979;57: 825-831

16. Varvarigou A, Bardin CL, Beharry K, Chemtob S, Papageorgiou A, Aranda JV. Early ibuprofen administration to prevent patent ductus arteriosus in premature newborn infants. JAMA 1996;275:539-544

17. Van Overmeire B, Follens I, Hartmann S, Creten WL, Van Acker KJ. Treatment of patent ductus arteriosus with ibuprofen. Arch Dis Child Fetal Neonatal Ed 1997;76: F179-184

18. Mosca F, Bray M, Lattanzio M, Fumagalli M, Tosetto C. Comparative evaluation of the effect of indomethacin and ibuprofen on cerebral perfusion and oxygenation in preterm infants with patent ductus arteriosus. J Pediatr 1997;131: 549-554

19. Grosfeld JL, Kamman K, Gross K, et al. Comparative effects of indomethacin, prostaglandin $\mathrm{E} 1$ and ibuprofen on bowel ischemia. J Pediatr Surg 1983;18:738-742

20. Malcom DD, Segar JL, Robillard JE, Chemtob S. Indomethacin compromises hemodynamics during positive-pressure ventilation independently of prostanoids. J Appl Physiol 1993;74:1672-1678

21. Patel J, Roberts I, Azzopardi D, Hamilton P, Edwards AD. Randomized double-blind controlled trial comparing the effects of ibuprofen with indomethacin on cerebral hemodynamics in preterm infants with patent ductus arteriosus. Pediatr Res 2000;47:36-42

22. Pezzati M, Vangi V, Biagiotti R, Bertini G, Cianciulli D, Rubatelli FF. Effects of indomethacin and ibuprofen on mesenteric and renal blood flow in preterm infants with patent ductus arteriosus. J Pediatr 1999;135:733-738

23. Shah SS, Ohlsson A. Ibuprofen for the prevention of patent ductus arteriosus in preterm and/or low birth weight infants. Cochrane Database Syst Rev 2006;(1):CD004213. DOI: 10.1002/14651858.CD004213.pub2

24. Van Overmeire B, Allegaert K, Casaer A, et al. Prophylactic ibuprofen in premature infants: a multicentre, randomized, double-blind, placebo-controlled trial. Lancet 2004;364: 1945-1949

25. Van Overmeire B, Smets K, Lecoutere D, et al. A comparison of ibuprofen and indomethacin for closure of patent ductus arteriosus. N Engl J Med 2000;343:674-681

26. Fanaroff AA, Stoll BJ, Wright LL, et al. Trends in neonatal morbidity and mortality for very low birthweight infants. Am J Obstet Gynecol 2007;196:147.e1-e8

27. Koch J, Hensley G, Roy L, Brown S, Ramaciotti C, Rosenfeld CR. Prevalence of spontaneous closure of the ductus arteriosus in neonates at a birth weight of 1000 grams or less. Pediatrics 2006;117:1113-1121

28. Gonzalez A, Sosenko IR, Chandar J, et al. Influence of infection on patent ductus arteriosus and chronic lung disease in premature infants weighing 1000 grams or less. J Pediatr 1996;128:470-478

29. Martin JA, Hamilton BE, Sutton PD, et al. Births: Final data for 2005. National vital statistics reports; Vol. 56, No. 6 . Hyattsville, MD: National Center for Health Statistics; 2007

30. Australian New Zealand Clinical Trials Registry. Targeting of treatment of the patent ductus arteriosus using early echocardiography (DETECT). ID\#: ACTRN 12608000295347. Available at: http://www.anzctr.org.au/ trial_view.aspx? $\mathrm{ID}=82886$. Accessed August 6, 2008 
31. Efron B. Forcing a sequential experiment to be balanced. Biometrika 1971;58:403-417

32. Aranda JV, Varvarigou A, Beharry K, et al. Pharmacokinetics and protein binding of intravenous ibuprofen in the premature newborn infant. Acta Paediatr 1997;86:289-293

33. Bell MJ. Neonatal necrotizing enterocolitis. N Engl J Med 1978;298:281-282

34. Gournay V, Roze JC, Kuster A, et al. Prophylactic ibuprofen versus placebo in very premature infants: a randomised, double-blind, placebo-controlled trial. Lancet 2004;364: 1939-1944

35. Chemtob S, Beharry K, Rex J, Varma DR, Aranda JV. Prostanoids determine the range of cerebral blood flow autoregulation of newborn piglets. Stroke 1990;21:777-784

36. Dani C, Bertini G, Pezzati M, et al. Prophylactic ibuprofen for the prevention of intraventricular hemorrhage among preterm infants: a multicenter, randomized study. Pediatrics 2005;115:1529-1535

37. Ment LR, Oh W, Ehrenkranz RA, et al. Low-dose indomethacin therapy and extension of intraventricular hemorrhage: a multicenter randomized trial. J Pediatr 1994; 124:951-955

38. Papile LA, Burstein J, Burstein R, Koffler H. Incidence and evolution of subependymal and intraventricular hemorrhage: a study of infants with birth weights less than 1,500 gm. J Pediatr 1978;92:529-534

39. Barkovich AJ, ed. Pediatric Neuroimaging. 3rd Ed. Philadelphia: Lippincott Williams and Wilkins; 2000:180-4

40. Thomas RL, Parker GC, Van Overmeire B, Aranda JV. A meta-analysis of ibuprofen versus indomethacin for closure of patent ductus arteriosus. Eur J Pediatr 2005;164:135-140

41. Romagnoli C, De Carolis MP, Papacci P, et al. Effects of prophylactic ibuprofen on cerebral and renal hemodynamics in very preterm neonates. Clin Pharmacol Ther 2000;67:676683

42. Gournay V, Savagner C, Thiriez G, Kuster A, Rozé JC. Pulmonary hypertension after ibuprofen prophylaxis in very preterm infants. Lancet 2002;359:1486-1488

43. Hasan J, Beharry K, Gharraee Z, et al. Early postnatal ibuprofen and indomethacin effects in suckling and weanling rat kidneys. Prostaglandins Other Lipid Mediat 2008;85:8188

44. Schmidt B, Roberts R, Davis $\mathrm{P}$, et al. The International Caffeine for Apnea of Prematurity (CAP) Trial. Preliminary analysis of outcome at corrected age of 18 to 21 months. Pediatric Academic Societies Meeting; May 5, 2007. Abstract 5130.1, Toronto, Canada

45. Kinsella JP, Cutter GR, Walsh WF, et al. Early inhaled nitric oxide therapy in premature newborns with respiratory failure. N Engl J Med 2006;355:354-364

46. Edwards AD, Tan S. Perinatal infections, prematurity and brain injury. Curr Opin Pediatr 2006;18:119-124

47. Dammann O, Leviton A. Inflammation, brain damage and visual dysfunction in preterm infants. Semin Fetal Neonatal Med 2006;11:363-368

\section{APPENDIX 1}

List of participating institutions and site investigators (The IV Ibuprofen Clinical Trials Group):

- University of Texas Southwestern Medical Center, Dallas, TX: Blaire Cox, M.D. (deceased), Charles Rosenfeld, M.D.

- Univ Hosp Antwerp, Belgium: Bart Van Overmeire, M.D., Ph.D., Pat Van Reempts, M.D., Ph.D.

- Sharp Mary Birch Hospital, University of California PPRU, San Diego, CA: Paul Wozniak, M.D.

- University of Miami, Jackson Memorial Hospital, Miami, FL: Ilene Sosenko, M.D.

- University of Alabama, Birmingham, AL: Waldemar Carlo, M.D., Robert Schelonka, M.D.

- Children's Hospital of Michigan, Wayne State University, Detroit, MI: Virginia Delaney-Black, M.D., Ron Thomas, Ph.D., J. Parker, G. Steinhilber

- Children's Hospital of New York City, New York, NY: Richard Polin, M.D.

- University of Utah Medical Center, Salt Lake City, UT: Robert Ward, M.D.

- Dornbecher Children's Hospital/OHSU, Portland, OR: Linda Wallen, M.D.

- New York Medical College Westchester Medical Center, Westchester, NY: Lance Parton, M.D.

- Winthrop University Hospital, Mineola, NY: Jonathan Davis, M.D., Linda Genen, M.D., Carolyn Levine, M.D., Donna Better, M.D., Carlos Montoya, M.D.

- Ross Abbott IV Ibuprofen Trial Team, Columbus, $\mathrm{OH}$ : Kevin Mahan, Ph.D., Alan Ryan, Ph.D., Robert Shalwitz, M.D., Gregory Gomez, Anand Seth, Ph.D., Geraldine Baggs, Ph.D., Jeffery Oliver, M.S., Susan Toth, M.P.H., Linna Liu, M.S., Sue Zhang, M.S., Nel VanLommel, Mark Poulson, Kelly Samaie, Veronica Knox, Mary Ann Cockram, Jerry Young, M.P.H., Charles Paule, M.S., Kristen Deluca, R.D., L.D., M.S., and Bobbie Swearengin, R.N.

- Data Safety Monitoring Committee: Ronald Clyman, M.D. (UCSF), Yvette Johnson, M.D., M.P.H. (Baylor) (Chair), Leonard Weissman, M.D. (Baylor); Stanley Lemeshow, Ph.D. (OSU)

The NICHD Pediatric Pharmacology Research Unit Network (NICHD PPRU), Children's Hospital of Michigan and Wayne State University, Detroit, MI, and the Intravenous Ibuprofen Clinical Trial Group. 